\title{
ACE Inhibitor-induced Dry Cough and Dysgeusia: COVID-19 Symptom Mimickers-A Case Series
}

\author{
Shambo S Samajdar ${ }^{1}$, Dipan Saha ${ }^{2}$, Satyabrata Sahoo ${ }^{3}$, Sumalya Sen ${ }^{4}$, Santanu K Tripathi ${ }^{5}$
}

\begin{abstract}
An outbreak of pneumonia caused by severe acute respiratory syndrome coronavirus 2 (SARS-CoV-2) that started in China in the year 2019 has become a global pandemic. SARS-CoV-2 enters the host cell through the angiotensin-converting enzyme 2 (ACE2) receptor, which is known to be down-regulated in COVID-19 infected individuals. ACE inhibitors (ACEI) and angiotensin receptor blockers (ARB) can modulate and most probably up-regulate ACE2 expression and can be a therapeutic option, especially in COVID-19 patients with hypertension. ACEl itself can cause dry cough and loss of taste in some susceptible individuals, which can intimate the symptoms of COVID-19 infection itself and can put the treating physician into a diagnostic dilemma. Careful evaluation and discontinuation of the drug can reverse the symptoms dramatically. Keywords: Angiotensin-converting enzyme 2, Angiotensin receptor blockers, COVID.

Bengal Physician Journal (2021): 10.5005/jp-journals-10070-7042
\end{abstract}

\section{INTRODUCTION}

In the latter half of 2019, some subjects with pneumonia of unknown cause were found to be infected with a novel coronavirus, known as coronavirus disease-2019 (COVID-19), in Hubei province, China, which had previously not been detected in humans or animals. ' Since then, the world has seen a COVID-19 pandemic in almost every continent across the globe. Studies involving confirmed COVID-19 cases have demonstrated age, concomitant diseases, and ethnicity as potentially important risk factors for susceptibility to hospitalization or death due to the complication of the disease. In addition, longterm use of some medications by the exposed individuals has been suggested as a potential risk factor for infection or severe adverse outcomes due to infection. ${ }^{2,3}$ Severe acute respiratory syndrome coronavirus 1 (SARS-CoV-1) and SARS-CoV-2, which are responsible for the SARS epidemic and the COVID-19 pandemic, respectively, interface with the renin-angiotensin-aldosterone system (RAAS) through angiotensin-converting enzyme 2 (ACE2), an enzyme that modulates the effects of the RAAS and is also the primary binding site for both SARS viruses. The interaction between these viruses and ACE2 may be one of the determinant factors of their infectivity, and there is some evidence that RAAS inhibitors may change ACE2 expression and hence COVID-19 virulence. Many researchers have reviewed this hypothesis. It has been observed that SARS-CoV-2 down-regulates ACE2. SARS-CoV-2 decreases ACE2 that is reversed by angiotensin receptor blockers (ARB) treatment. There is also little evidence that treatment with angiotensin-converting enzyme inhibitors (ACEI) and ARBs can decrease the severity of pulmonary injury by SARS-CoV-2.There is no study to discontinue ACEI or ARBs in SARS-CoV-2 patients having hypertension. ${ }^{4}$

On the contrary, ACEl and ARB have been used for many years in the treatment of hypertension and cardiovascular diseases. In addition to its antihypertensive effects, beneficial effects on mortality and complications of diseases, such as coronary artery disease, heart failure, diabetes, and diabetic nephropathy, have also been documented. ${ }^{5}$ Although they are generally well-tolerated, some of the patients discontinue their treatments due to side effects, especially cough and rarely dysgeusia. ${ }^{6}$ During the recent SARS-CoV pandemic, it has
${ }^{1-5}$ Department of Clinical and Experimental Pharmacology, School of Tropical Medicine, Kolkata, West Bengal, India

Corresponding Author: Shambo S Samajdar, Department of Clinical and Experimental Pharmacology, School of Tropical Medicine, Kolkata, West Bengal, India, e-mail: Shambo_sa2001@yahoo.co.in

How to cite this article: Samajdar SS, Saha D, Sahoo S, et al. ACE Inhibitor-induced Dry Cough and Dysgeusia: COVID-19 Symptom Mimickers-A Case Series. Bengal Physician Journal 2021;8(2):49-51.

Source of support: Nil

Conflict of interest: None

not been uncommon for clinicians to come across patients who are hypertensive and have been on ACEI or ARB class of drugs. During treatment, if these patients develop cough and loss of taste, it is very difficult for clinicians to distinguish whether symptoms are due to the adverse effect of drugs or the patient has been infected by the SARS$\mathrm{CoV}$. In this article, we will discuss four such cases with a similar scenario.

\section{Case Descriptions}

\section{Case 1}

A 46-year-old bank worker who is a known case of hypertension and type 2 diabetes mellitus (DM) for the last 2 years presented at the Clinical Pharmacology and Therapeutics Clinic of School of Tropical Medicine, Kolkata. He was on tablet enalapril $5 \mathrm{mg}$, atorvastatin $20 \mathrm{mg}$, tablet teneligliptin, metformin fixed-dose combination (FDC), and pantoprazole 40. Prior to enalapril, he was on tablet amlodipine $5 \mathrm{mg}$. He developed pedal edema for which he consulted his treating physician who stopped amlodipine and started enalapril 5 mg on June 6, 2020. On July 12,2020, he turned up at the outpatient department (OPD) once again with complaints of dry cough and tastelessness, as his coworker was COVID-19 positive, he insisted me to do a COVID-19 test. After 3 days, he informed me that the reverse transcription-polymerase chain reaction (RT-PCR) test for SARS-CoV-2 was found negative. I then 
deprescribed enalapril 5 and put him on benidipine $8 \mathrm{mg}$. In addition, the patient was advised to take some cough suppressant. Cough reduced after few days. On 31st July, he consulted me over the telephone that there was no cough for the last 2 weeks and alteration of taste has also been reversed.

\section{Case 2}

Mrs. TRC a 48-year-old female came to clinical pharmacology OPD with the chief complaints of cough and loss of taste (dysgeusia) on August 9, 2020. She is a known case of hypertension and type 2 DM. She had been on perindopril (4 mg), amlodipine (5 mg), glimepiride $2 \mathrm{mg}$, and metformin $500 \mathrm{mg}$ from July 24, 2020. As it was a COVID-19 pandemic situation, she was advised to do RT-PCR nasopharyngeal swab test for SARS-CoV-2, but it was found negative. So, possibly these symptoms were due to perindopril as ACEI have a more chance of causing cough and dysgeusia, and I deprescribed perindopril on August 12,2020, and prescribed tablet chlorthalidone (12.5). On August 30, 2020, she reported being free of all respiratory symptoms.

\section{Case 3}

Mr. RM a 60-year-old male came to clinical pharmacology OPD on June 12, 2021, with a chief complaint of dry cough. He was a known case of hypertension, ischemic heart disease, and obstructive airway disease. He was on FDCs of perindopril (4) + indapamide (1.5) + amlodipine (5). Apart from that, he was on DPI of fluticasone and salmeterol and oral rosuvastatin and clopidogrel FDC from May 20, 2021. He was advised to do RT-PCR nasopharyngeal swab test for SARS-CoV-2, but it was found negative. On June 20, 2021, I stopped the FDC containing perindopril 80 and put him on amlodipine, cardioselective beta-blocker, and chlorthalidone, and then he cured of symptoms on July 12, 2021.

\section{Case 4}

Mrs. JS a 78-year-old female came to clinical pharmacology OPD on August 19,2020, with the chief complaints of cough and loss of taste. She was on FDCs of enalapril and amlodipine from August 7, 2020. After getting the nasopharyngeal swab test for SARS-CoV-2 done on August 21,2020, the above-mentioned FDC was discontinued as the result was found to be negative; she was given CTD (12.5), and on September 12, 2020, she got cured of her symptoms.

\section{Discussion}

Cough, along with fever and fatigue, is one of the key symptoms of COVID-19. This virus uses ACE2 as a source of cellular entry, as do the SARS-CoV-1 and virus known to have caused Middle East respiratory syndrome. ACE2 is predominantly found in lung alveolar cells, bronchial epithelium, and vascular endothelium, explaining why the respiratory tract and lung serve as a primary site of viral entry. ${ }^{8}$ Proinflammatory cytokines like interleukin- 6 are also elevated, similar to that seen in sepsis-induced ARDS in general. ${ }^{9}$ It is having an incubation period of 4-7 days generally. Symptoms include cough, shortness of breath, sore throat, rhinorrhea, headache, anosmia, diarrhea, nausea, and vomiting. Among the symptoms, fever, cough, and shortness of breath are the commonest. ${ }^{10}$ It is understood that subunits of spike (S) proteins of SARS-CoV-2 and SARS-CoV have an affinity toward human ACE2 receptors. ${ }^{11}$ ACE2 mediates the conversion of angiotensin II (Ang II) to Ang (1-7) and Ang I to Ang (1-9). ACE2 is a homolog of the ACE and is found in cell membranes of the heart, kidney, lungs, arteries, and intestines. It has been observed that SARS-CoV-2 binds to the ACE2 receptor, 10-20-folds higher compared to SARS-CoV, resulting in higher infection and transmissibility. ${ }^{12}$ ACE2 helps to protect lungs from severe acute lung injury and kidneys from damage. The deficiency of ACE2 is related to glomerulosclerosis, oxidative stress in the renal system, and diabetic nephropathy. ACE2 expression is decreased during SARS-CoV infections, resulting in the change of dynamic equilibrium state between ACE/Ang II/AT1R axis and ACE2/Ang (1-7)/Mas receptor axis, and similarly this likely happens during SARS-CoV-2 infection. As SARS-CoV-2 gets into the host cell through ACE2 receptors ${ }^{13}$ and then downregulates its expression such that ACE2 losses its protective effect in different organs, activating ACE2 may lead to the treatment for COVID-19. As the active sites of ACE and ACE2 are different, ACEI does not directly affect ACE2 activity but can cause activation of ACE2. ACEI like captopril activates ACE2/ Ang (1-7) level/Mas receptor axis and lowers mean pulmonary arterial pressure and right ventricular index. ${ }^{14}$ Till date, there is no confirmed treatment option for COVID-19, and drugs activating ACE2 like ACEl and ARBs can be beneficial for the COVID-19 patients, especially who are presented with comorbid hypertension or ischemic cardiomyopathy. As it has been discussed earlier, patients taking these class of drugs may experience new onset of dry cough and loss of taste, which can imitate the symptoms of COVID-19 itself. In those cases, careful evaluation and stoppage of the drug may improve the symptoms dramatically.

\section{Conclusion}

ACEI class of drugs either alone or in combination may be repurposed to upregulate the host ACE2, thereby serving as probable SARS-CoV-2 therapeutics. These drugs may enhance the immune responses, reduce inflammation, and end-organ damage due to COVID-19 complications. Careful clinical evaluation should be done if the patient develops symptoms that mimic symptoms of COVID-19 pneumonitis in this pandemic time. The ADR due to ACEl was categorized as probable according to WHO-UMC Scale and Naranjo ADR Probability Scale. Severe ADRs can be prevented if we diagnose early and treat them immediately. Prompt recognition of ADRs of drugs can prevent the economic burden of COVID-19 tests.

\section{References}

1. WHO. Clinical management of severe acute respiratory infection when Novel coronavirus ( $\mathrm{nCoV}$ ) infection is suspected: interim guidance. Available at: https://www.who.int/publications-detail/ clinical-management-of-severe-acute-respiratory-infectionwhennovel-coronavirus-(ncov)-infection-is-suspected (accessed 11 January 2020)

2. Garg S, Kim L, Whitaker M, et al. Hospitalization rates and characteristics of patients hospitalized with laboratory-confirmed coronavirus disease 2019 - COVID-NET, 14 states, March 1-30, 2020. MMWR Morb Mortal Wkly Rep 2020;69:458-464. DOI: 10.15585/ mmwr.mm6932e3.

3. Zhang P, Zhu L, Cai J, et al. Association of inpatient use of angiotensin-converting enzyme inhibitors and angiotensin II receptor blockers with mortality among patients with hypertension hospitalized with COVID-19. Circ Res 2020;126:1671-1681.DOI: 10.1161/ CIRCRESAHA.120.317134.

4. Gheblawi M, Wang K, Viveiros A, et al. Angiotensin-converting enzyme 2: SARS-CoV-2 receptor and regulator of the reninangiotensin system: celebrating the 20th anniversary of the discovery of ACE2. Circ Res 2020;126(10):1456-1474. DOI: 10.1161/ CIRCRESAHA.120.317015.

5. Chobanian AV, Bakris GL, Black HR, et al. The seventh report of the joint national committee on prevention, detection, evaluation, 
and treatment of high blood pressure: the JNC 7 report. JAMA 2003;289:2560-2572. DOI: 10.1001/jama.289.19.2560.

6. Morimoto T, Gandhi TK, Fiskio JM, et al. An evaluation of risk factors for adverse drug events associated with angiotensin-converting enzyme inhibitors. J Eval Clin Pract 2004;10:499-509. DOI: 10.1111/j.13652753.2003.00484.x.

7. WHO.Q\&A on coronaviruses (COVID19). 2020. Available at: https://www. who.int/emergencies/diseases/novelcoronavirus-2019/question-andanswers-hub/q-a-detail/qa-coronaviruses (accessed 17 November 2020)

8. Huang $C$, Wang $Y, L i X$, et al. Clinical features of patients infected with 2019 novel coronavirus in Wuhan, China. Lancet 2020;395:497-506. DOI: 10.1016/S0140-6736(20)30183-5.

9. Englert JA, Bobba C, Baron RM. Integrating molecular pathogenesis and clinical translation in sepsis-induced acute respiratory distress syndrome. JCl Insight 2019;4:1-13. DOI: 10.1172/jci.insight.124061.

10. Chen N, Zhou M, Dong X, et al. Epidemiological and clinical characteristics of 99 cases of 2019 novel coronavirus pneumonia in
Wuhan, China: a descriptive study. N Engl J Med 2020;395:507-513. DOI: 10.1016/S0140-6736(20)30211-7.

11. Zhou P, Yang X-L, Wang X-G, et al. A pneumonia outbreak associated with a new coronavirus of probable bat origin. Nature 2020;579:270273. DOI: 10.1038/s41586-020-2012-7.

12. Wrapp D, Wang N, Corbett KS, et al. Cryo-EM structure of the 2019nCoV spike in the prefusion conformation. Science 2020;367:12601263. DOI: 10.1126/science.abb2507.

13. Kuba $K$, Imai $Y$, Rao $S$, et al. A crucial role of angiotensin converting enzyme 2 (ACE2) in SARS coronavirus-induced lung injury. Nat Med 2005;11:875-879. DOI: 10.1038/nm1267.

14. Li Y, Zeng Z, Li Y, et al. Angiotensin-converting enzyme inhibition attenuates lipopolysaccharide-induced lung injury by regulating the balance between angiotensin-converting enzyme and angiotensin-converting enzyme 2 and inhibiting mitogen-activated protein kinase activation. Shock 2015;43:395-404. DOI: 10.1097/ SHK.0000000000000302. 